\title{
Battling the biology of opioid addiction
}

\author{
Deaths from drug overdose are rising worldwide, in part owing to the growing epidemic of opioid addiction. Efforts \\ to combat opioid addiction will benefit from stronger collaboration between preclinical researchers who are studying \\ addiction and those studying chronic pain.
}

W orldwide, more than 29 million people experience drug addiction or have drug-use disorders, resulting in more than 200,000 drug-related deaths in 2014. Although the prevalence of drug use and drug addiction have remained stable over the past decade, at $5 \%$ and $0.6 \%$, respectively, drug-related deaths are on the rise. Annual deaths from drug overdose have risen steadily, from approximately 30,000 per year in 2005 to more than 50,000 per year in 2015 in the United States. More troublingly, recent estimates point to an additional $19 \%$ increase for 2016. The phenomenon is global. In Canada, British Columbia alone witnessed a $140 \%$ increase from 210 deaths in 2010 to more than 500 in 2015 , and an $80 \%$ annual increase was recently reported for 2016. From 2004-14, drug-related deaths rose by $44 \%$ in the United Kingdom, surpassing 2,200 per year. And in Europe, there have been 6\% more drug-overdose-related deaths in 2015 as compared to the previous year, continuing a trend in which deaths from overdose have risen for each of the past three years.

Although multiple factors are responsible for this increase, the contribution of opioid addiction and abuse cannot be ignored. From 2014-15, opioid overdoses accounted for $60 \%$ of the US drugoverdose mortality rate, totaling more than 32,000 deaths (MMWR Morb. Mortal. Wkly. Rep. 65, 1445-1452, 2016). According to the US National Institute on Drug Abuse (NIDA), it is estimated that as many as 90 people in the US die each day from opioid overdose. Opioidoverdose deaths in the United States have already surpassed the annual mortality associated with prostate cancer $(27,000$ per year), and, if trends continue, opioid overdoses might soon claim as many lives as those lost to breast cancer (approximately 40,000 per year, according to estimates by the US Centers for Disease Control and Prevention). One of the factors contributing to the opioid-addiction epidemic is the transition from the use of prescription opioid-based painkillers for the treatment of moderate or severe chronic pain (such as postoperative pain) to their misuse or abuse, leading to subsequent addiction. According to NIDA, approximately $10 \%$ of patients prescribed opioid painkillers develop an opioid-use disorder, and half of these individuals progress to heroin use.

In late May, NIDA Director Nora Volkow and US National Institutes of Health (NIH) Director Francis Collins published a Special Report in the New England Journal of Medicine outlining major strategic initiatives intended to focus opioid-addiction research on three areas (N. Engl. J. Med., http://doi.org/b8sf, 2017). The first of these seeks to investigate new therapies that might directly treat opioid overdose acutely, for instance, by improving delivery methods and the potency of opioid-receptor antagonists such as naloxone. The other two aims call for investigation into fundamentally new therapies that target the underlying neurobiology of opioid addiction and chronic pain. A subtle yet crucial point to add is that a better understanding of the pathophysiology of chronic pain and its intersection with opioid receptors and reward circuitry will be required to identify novel targets for future therapeutic intervention. Stronger collaboration between investigators in the addiction and pain fields will be an essential component of any successful effort to stem the tide of opioid addiction.

Collaborative efforts to facilitate the integration of addiction and pain research have the potential to uncover novel processes that might not be evident when studying each of these phenomena in isolation. For example, changes in the brain's reward circuitry that are caused by chronic opioid self-administration might be fundamentally different when initiated in the context of a chronic-pain model or after previous exposure to opioids to treat chronic pain. Similarly, neurobiological processes controlling withdrawal behaviors or relapse risk are likely to be modified by an underlying chronic pain condition. Given that other neuropsychiatric conditions can also increase risk for drug addiction in humans, it will be valuable to begin assessing whether addiction biology or response to treatment differs under conditions of stress or in animal models of anxiety or depression.

With regard to animal models, a key challenge will be transitioning to endpoints that are more relevant to the human experience of pain and addiction. The most common behavioral endpoints typically used to assess novel analgesic efficacy in animal models of chronic pain are relatively simple measures of reflexive nociceptive behaviors, which typically don't take into account other dimensions of spontaneous or evoked 'pain' behaviors that engage the brain's reward circuitry. Encouragingly, pain researchers have begun to incorporate assays that quantify changes in motivation, reward and aversion, or negative-affect and depressive behaviors that might be more similar to what is experienced in human populations experiencing chronic pain. Efforts to understand the neurobiological processes that contribute to addiction will be aided by the use of tractable preclinical models that more closely resemble the course of human addiction. These preclinical addiction models need to take into account not only initial exposure and selfadministration of opioids, but also abstinence, withdrawal and eventual relapse. Each of these complicated phenomena probably arises from a distinct neurobiological process, and it follows then that these processes might need to be engaged by different therapeutic targets.

Pain relief itself is a naturally rewarding condition. Successfully grappling with the connections between pain and reward will require embracing efforts to connect the pain and reward research communities. 